\title{
The Value of Cerebral CT Angiography with Low Tube Voltage in Detection of Intracranial Aneurysms
}

\author{
Kun Tang, ${ }^{1}$ Rui Li, ${ }^{1}$ Jie Lin, ${ }^{1}$ Xiangwu Zheng, ${ }^{1}$ Ling Wang, ${ }^{1,2}$ and Weiwei Yin ${ }^{1}$ \\ ${ }^{1}$ Department of Radiology, The First Affiliated Hospital of Wenzhou Medical University, Wenzhou, Zhejiang 325000, China \\ ${ }^{2}$ Department of Nuclear Medicine, Wenzhou Medical University, Wenzhou, Zhejiang 325000, China \\ Correspondence should be addressed to Ling Wang; laohu9823@163.com
}

Received 8 October 2014; Revised 9 December 2014; Accepted 9 December 2014

Academic Editor: Carl Muroi

Copyright (c) 2015 Kun Tang et al. This is an open access article distributed under the Creative Commons Attribution License, which permits unrestricted use, distribution, and reproduction in any medium, provided the original work is properly cited.

\begin{abstract}
Objective. The aim of this study is to investigate the value of cerebral CT angiography (CTA) with low tube voltage in detection of intracranial aneurysms. Materials and Methods. A total of 294 consecutive patients with spontaneous subarachnoid hemorrhage (SAH) were enrolled in this study and randomly assigned into conventional voltage CTA (C-CTA) group and low voltage CTA (LCTA) group. The objective and subjective image qualities were analyzed and compared between C-CTA and L-CTA groups. With the results of 3D-DSA as "gold standard," the sensitivity, specificity, and accuracy of C-CTA and L-CTA in diagnosis of aneurysms were calculated and compared with each other. Results. Compared with group C-CTA, the CT dose index volume (CTDIvol) of group L-CTA reduced by $35.65 \%$. There were no significant differences between C-CTA and L-CTA groups regarding objective and subjective image qualities. The sensitivity, specificity, and accuracy of L-CTA in diagnosis of aneurysms were $95.16 \%, 99.72 \%$, and 99.42\%, respectively. There were no significant differences in sensitivity, specificity, and accuracy between the C-CTA and L-CTA groups. Conclusion. The value of cerebral CTA with $100 \mathrm{kV}$ low tube voltage in detection of intracranial aneurysms is significant, and it should be recommended as a routine scan method.
\end{abstract}

\section{Introduction}

Spontaneous subarachnoid hemorrhage (SAH) is caused by rupture of an intracranial aneurysm in $80-90 \%[1,2]$ of cases. The mortality for untreated aneurismal SAH is up to $43-$ $67 \%[3-5]$ in the first month. Therefore, early diagnosis and early treatment are the most effective way to prevent the death of patients. Recently, computed tomography angiography (CTA) technology is playing an increasing role in the screening of patients suspected of having intracranial aneurysms, but it also has the potential to lead to an increase in radiation dose owing to the routine use of thinner sections and the extended volume of acquisition. Although magnetic resonance angiography (MRA) is an alternative nonradiation technique in diagnosis of intracranial aneurysms, the main disadvantages of this technique are the prolonged acquisition time and the artifacts due to flow phenomena and patient motion. A recent meta-analysis indicates that sensitivity of MRA has become comparable with that of CTA in the diagnosis of intracranial aneurysms, but specificity of MRA seems inferior compared with CTA [6]. Therefore, CTA has widely been used as a routine primary tool in diagnosis of intracranial aneurysms, and the concern regarding radiation dose in CT scan has been increasing, especially the potential harm to the brain [7].

However, there are few reports regarding low radiation dose during neuroimaging, especially the CTA with low tube voltage in detection of intracranial aneurysms. Most of the available studies using low dose CTA focus on great vessels, such as carotid artery [8] and pulmonary artery [9]. Even in those articles referring to neurovascular imaging with low tube voltage, the results were inconsistent and the cases involved in those studies were limited [10-13]. In this study, a large sample was enrolled and assigned into conventional voltage CTA (C-CTA) group and low voltage CTA (L-CTA) group. The aim of this study is to investigate the value of CTA with low tube voltage in detection of intracranial aneurysms by comparing image qualities, radiation dose, and detection accuracy of aneurysms between C-CTA group and L-CTA group, with three-dimensional digital subtraction angiography (3D-DSA) as the reference standard. 


\section{Materials and Methods}

The institutional review board approved this study, and informed consent was obtained from all participating patients.

2.1. Patients. Between October 2010 and May 2012, a total of 294 consecutive patients (130 males, 164 females; mean age $56.89 \pm 13.30$ years) with $\mathrm{SAH}$ were enrolled in this prospective study. The inclusion criteria were that patients have clinical evidence of intracranial aneurysms and are able to undergo both CTA and DSA. The exclusion criteria were history of allergy to iodine-containing contrast medium, serious cardiac renal insufficiency, and pregnancy.

The patients who were enrolled in this study were randomly assigned into C-CTA group and L-CTA group. The CCTA group included 148 patients ( 64 males, 84 females; mean age $56.66 \pm 13.02$ years) and the L-CTA group included 146 patients ( 66 males, 80 females; mean age $57.13 \pm 13.62$ years). All of the patients underwent both 64-slice CTA and 3D-DSA within 3 days successively.

\subsection{Study Protocols}

2.2.1. CTA Protocol. All imaging procedures were performed using a 64-row multislice CT system (GE LightSpeed, 64-row multislice CT). The CTA was initiated 18-20 seconds after the start of infusion of nonionic iodinated contrast material (iohexol $350 \mathrm{mgI} / \mathrm{mL}$ ) from dorsal vein or antecubital vein. Nonionic iodinated contrast material was injected with a powered injector at a rate of $4 \mathrm{~mL} / \mathrm{s}$. The volume of nonionic iodinated contrast material in each study was generally 60$80 \mathrm{~mL}$. Scanning range is from basis cranii to calvaria.

The CTA data acquisition was performed according to the following protocol: tube voltage of $120 \mathrm{kV}$ (C-CTA) or $100 \mathrm{kV}$ (L-CTA); tube current of $380 \mathrm{~mA}$; slice thickness of $5.0 \mathrm{~mm}$; section interval of $5.0 \mathrm{~mm}$; collimation of $64 \times$ $1.25 \mathrm{~mm}$; rotation time of $0.6 \mathrm{~s}$; pitch of 0.562 ; FOV of $25 \mathrm{~cm}$; matrix of $512 \times 512$; reconstruction thickness of $1.25 \mathrm{~mm}$; and reconstruction interval of $0.6 \mathrm{~mm}$.

The review of the CT images was processed by a workstation (ADW Workstation 4.3) to acquire MRP, MIP, and VR algorithms in each case.

2.2.2. DSA Protocol. All DSA examinations were performed with femoral catheterization by Seldinger technique with a DSA system (Philips Allura Xper FD20, Netherlands). Typically, nonionic iodinated contrast material (iohexol $350 \mathrm{mgI} / \mathrm{mL}$ ) was used in all cases. Standard injection rates and volumes were as follows: $5 \mathrm{~mL} / \mathrm{s}$ for $8 \mathrm{~mL}$ for common carotid artery and $4 \mathrm{~mL} / \mathrm{s}$ for $7 \mathrm{~mL}$ for vertebral artery. The parameters were as follows: rotation angle: 0-2400; rotational speed: 550/s; picking rate: 30 frames/s; image matrix: $1024 \times$ 1024; image reconstruction matrix: $256 \times 256$; and exposure lag: $2 \mathrm{~s}$. The $3 \mathrm{D}-\mathrm{DSA}$ injection rates and volumes were as follows: $5 \mathrm{~mL} / \mathrm{s}$ for $25 \mathrm{~mL}$ for common carotid artery and $3 \mathrm{~mL} / \mathrm{s}$ for $12 \mathrm{~mL}$ for vertebral artery. The review of the DSA image data was processed by a workstation (Philips Integris
3D RA Release 4.3) to acquire 3D-DSA reconstructed images, such as VRD and SSD algorithms.

\subsection{Image Analysis}

2.3.1. Objective Image Quality of CTA. The CT attenuation values were measured on axial images using a manually defined circular region of interest (ROI) with diameter of 2-10 $\mathrm{mm}$. The measurement locations were as follows: basal artery (BA), left and right side of the internal carotid artery (ICA) of intracranial segment, the anterior cerebral artery (ACA), middle cerebral artery (MCA), and posterior cerebral artery (PCA). The CT values were measured three times at different segment of each vessel, using the average for the vascular CT value. Taking occipital lobe parenchyma (OLP) as vascular background, image noise is the standard deviation (SD) of the attenuation values of the background. All CTA images measurement was independently performed by an experienced radiologist.

Contrast-to-noise ratio (CNR) and signal-to-noise ratio $(\mathrm{SNR})$ are calculated as follows: $\mathrm{CNR}=(\mathrm{ROIa}-\mathrm{ROIb}) / \mathrm{SD}$; $\mathrm{SNR}=\mathrm{ROIa} / \mathrm{SD}$, where ROIa and ROIb are the CT numbers of the vessel region of interest and of the background region of interest, respectively.

2.3.2. Subjective Image Quality of CTA. For the subjective assessment of CTA image quality, two experienced observers who were blind to each set of scanning parameters were asked to evaluate all CTA images independently. The arterial margin, depiction of small arterial details, venous contamination, side-branches, adjacent perforators, the whole image quality, and the diagnostic confidence were assessed, respectively, by the use of a 5-point score system. The scoring criteria details are shown in Table 1.

2.4. Aneurysm Analysis. All DSA and CTA images were evaluated by consensus of two experienced neuroradiologists. The observation and analysis of the aneurysm on CTA and 3D-DSA were evaluated at 13 standard vessels [14, 15]: (1) the left and right vertebral artery of intracranial segment, (2) basilar artery, (3) posterior communicating artery, (4) left and right posterior cerebral artery, (5) left and right internal carotid artery of intracranial segment, (6) the left and right middle cerebral artery, (7) left and right anterior cerebral artery, and (8) anterior communicating artery.

If an aneurysm was considered present, the largest diameter and the size of the aneurysm neck of each aneurysm on CTA and 3D-DSA images were measured by one experienced neuroradiologist. The largest diameter of each aneurysm was measured and graded as $\geq 5 \mathrm{~mm}, 3-5 \mathrm{~mm}$, or $\leq 3 \mathrm{~mm}$. In addition, the anatomical relationship between aneurysm and adjacent structures and the feeding artery of the aneurysm was analyzed.

2.5. Radiation Dose. We used the CT dose index volume (CTDIvol, mGy) provided by the manufacturer for estimation of radiation dose. Effective dose (ED, mSv) was calculated according to dose-length product (DLP, $\mathrm{mGy} \cdot \mathrm{cm}$ ) and the tissue weighting factor $k\left(\mathrm{mSv} \times \mathrm{mGy}^{-1} \times \mathrm{cm}^{-1}\right)$ 
TABLE 1: Subjective image quality assessment.

\begin{tabular}{lccccc}
\hline Evaluation items & 5 points & 4 points & 3 points & 2 points & 1 point \\
\hline Arterial margin & Smooth & Mild rough & Moderate Rough & Rough & Extensive Rough \\
Depiction of small arterial details & Best & Better & General & Bad & Worse \\
Venous contamination & Least & Less & General & Much & More \\
Side-branches & Clear & Moderate clear & Mild clear & Rough & Unclear \\
Perforators & Clear visibility & Moderate visibility & Mild visibility & Hardly visible & Invisible \\
The whole image quality & Best & Better & General & Bad & Worse \\
Diagnostic confidence & Sufficient & Less sufficient & General & Insufficient & Unable to diagnose \\
\hline
\end{tabular}

commended by the European Commission (0.0021) [16]. The calculated formula is $\mathrm{ED}=k \times \mathrm{DLP}$.

2.6. Statistical Analysis. We used two-tailed Student's $t$-test to evaluate differences in scan length, image objective quality, and radiation dose between group C-CTA and group L-CTA. For subjective assessment, Wilcoxon's signed-rank test was used to analyze the differences between the two CTA groups. The sensitivity, specificity, and accuracy of diagnosis were calculated by using chi-square test. The largest diameter of aneurysm and the size of aneurysm neck in both CTA groups were compared with DSA using correlation analysis. Interobserver variation was assessed using Cohen kappa statistics. Kappa values less than 0.20 indicated poor agreement; $0.21-$ 0.40 , fair agreement; $0.41-0.60$, moderate agreement; $0.61-$ 0.80 , good agreement; and 0.81-1.00, excellent agreement. All statistical analyses were performed with a commercially available software package (SPSS, version 17.0) and a $P$ value of less than 0.05 was considered to be statistically significant.

\section{Results}

A total of 294 patients (130 males, 164 females) were enrolled in this study and underwent CTA and 3D-DSA within 3 days successfully. The average scan length of C-CTA and L-CTA was $16.73 \pm 1.35 \mathrm{~cm}$ and $16.59 \pm 1.03 \mathrm{~cm}$, respectively. The difference of scan length between the two groups was not statistically significant $(P=0.720)$.

3.1. Radiation Dose. The results of radiation dose including CTDIvol, DLP, and ED of group C-CTA and group LCTA are shown in Table 2. Compared with the group CCTA, the CTDIvol, DLP, and ED of group L-CTA were reduced by $35.65 \%, 35.27 \%$, and $35.35 \%$, respectively. The differences of average DLP and ED between the two groups were statistically significant $(P=0.000)$.

3.2. Image Quality Results. The mean vessel attenuation of group L-CTA was increased by approximately 19\%-22\% compared with the group C-CTA. The difference between the two groups was statistically significant $(P=0.000)$. At the same time, the contrast value between vessels and cerebral parenchyma at low voltage group was also substantially higher than that of standard voltage group $(P=0.000)$. Although the noise level was markedly higher at group LCTA, the calculated CNRs and SNRs showed no differences
TABLE 2: The comparative results of radiation dose between C-CTA and L-CTA groups.

\begin{tabular}{lcccc}
\hline $\begin{array}{l}\text { Radiation } \\
\text { dose }\end{array}$ & Group C-CTA & Group L-CTA & $t$ & $P$ \\
\hline $\begin{array}{l}\text { CTDIvol } \\
(\mathrm{mGy})\end{array}$ & 55.73 & 35.86 & $\ldots$ & $\ldots$ \\
\hline $\begin{array}{l}\text { Scan length } \\
(\mathrm{cm})\end{array}$ & $16.73 \pm 1.35$ & $16.59 \pm 1.03$ & 0.37 & 0.720 \\
$\begin{array}{l}\mathrm{DLP} \\
(\mathrm{mGy} \cdot \mathrm{cm})\end{array}$ & $932.55 \pm 112.35$ & $594.76 \pm 102.34$ & 18.24 & 0.000 \\
ED $(\mathrm{mSv})$ & $1.96 \pm 0.45$ & $1.25 \pm 0.39$ & 18.24 & 0.000 \\
\hline
\end{tabular}

between the two groups (CNRs: $P=0.509,0.596,0.833$, 0.933 , and 0.868 ; SNRs: $P=0.285,0.367,0.744,0.590$, and $0.737)$.

There were no significant differences between C-CTA and L-CTA groups regarding scores for arterial margin, depiction of small arterial details, venous contamination, side-branches, adjacent perforators, the whole image quality, and the diagnostic confidence $(P=0.121,0.325,0.233,0.112$, $0.233,0.413$, and 0.883 ). Using Cohen kappa statistics, the interobserver agreement in regard to subjective assessment of image quality was $0.77,0.73$ (kappa $>0.60$ ), which meant that the observation had good consistency and high reliability.

3.3. Aneurysm Diagnosis Results. In all 294 cases, a total of 273 aneurysms were identified by 3D-DSA in 243 patients. Solitary aneurysm was found in 215 cases, while multiple aneurysms were found in 28 cases, including 26 patients with two aneurysms and 2 patients with 3 aneurysms. The mean diameter of the aneurysms was $5.26 \pm 2.64 \mathrm{~mm}$ (range 1.5 to $18.3 \mathrm{~mm}$ ). The distribution of aneurysms according to the size was 123 of $\geq 5 \mathrm{~mm}$; 114 of 3 to $5 \mathrm{~mm}$; and 36 of $<3 \mathrm{~mm}$. The mean size of aneurysms neck was $2.99 \pm 1.14 \mathrm{~mm}$ (range 0.7 to $9.4 \mathrm{~mm}$ ).

Compared with the 3D-DSA reference standard, the accuracy of C-CTA and L-CTA in detection of intracranial aneurysms is shown in Table 3 and Figures 1 and 2. Three aneurysms were misdiagnosed and nine aneurysms were missed with C-CTA, while five aneurysms were misdiagnosed and six aneurysms were missed with L-CTA. Compared with 3D-DSA, the accuracy of C-CTA and L-CTA in diagnosis of aneurysms was not statistically different. Thesensitivity, specificity, and accuracy in the C-CTA and LCTA were $93.96 \%, 99.83 \%$, and $99.38 \%$ and $95.16 \%, 99.72 \%$, 
TABLE 3: Accuracy of C-CTA and L-CTA in the detection of intracranial aneurysms.

\begin{tabular}{|c|c|c|c|c|c|c|c|}
\hline Group & True positive & False positive & False negative & True negative & Sensitivity & Specificity & Accuracy \\
\hline  & 140 & 3 & 9 & 1777 & $93.96 \%$ & $99.83 \%$ & $99.38 \%$ \\
\hline L-CTA & 118 & 5 & 6 & 1771 & $95.16 \%$ & $99.72 \%$ & $99.42 \%$ \\
\hline
\end{tabular}



(a)

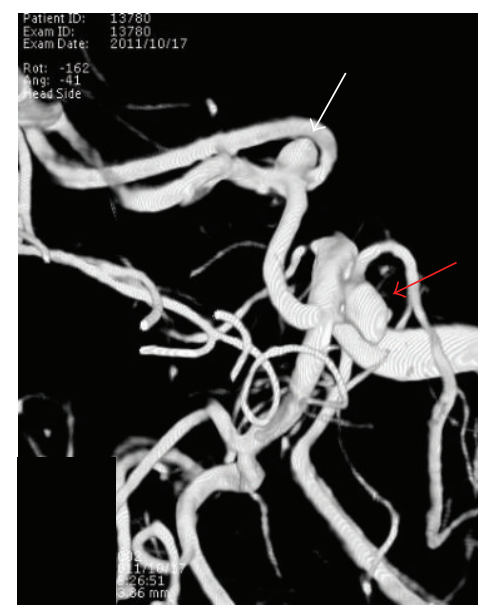

(b)

FIGURE 1: Images from a 61-year-old woman with SAH. L-CTA scanning shows an aneurysm ((a), white arrow) at anterior communicating artery and an aneurysm ((a), red arrow) at posterior communicating artery. 3D-DSA depicts the aneurysms at the same locations ((b), white and red arrows). The maximum diameter and neck sizes of anterior communicating artery aneurysm were $7.32 \mathrm{~mm}$ and $3.40 \mathrm{~mm}((\mathrm{~b})$, white arrow); and those of posterior communicating artery aneurysm were $5.44 \mathrm{~mm}$ and $3.86 \mathrm{~mm}$ ((b), red arrow).



(a)



(b)

FIgURE 2: Images from a 37-year-old woman with SAH. The L-CTA (a) and 3D-DSA (b) showed the same aneurysm at the same location. The maximum diameter of aneurysm and neck sizes in C-CTA (a) and 3D-DSA (b) were 4.5, $2.7 \mathrm{~mm}$ and 4.85, $2.57 \mathrm{~mm}$, respectively (red arrow).

and $99.42 \%$, respectively. There were no statistically significant differences between the two groups. For aneurysms of $\geq 5 \mathrm{~mm}, 3$ to $5 \mathrm{~mm}$, and $<3 \mathrm{~mm}$, the diagnostic results of $\mathrm{C}$ CTA and L-CTA were shown in Table 4. The differences of the two groups in detection of different size of aneurysms were not statistically significant $(P=1.0)$.

The mean maximum diameter and mean neck size of aneurysms in group C-CTA and group L-CTA were $5.53 \pm$
2.55 mm (95\% CI: 5.11-5.95), $2.97 \pm 1.24$ mm (95\% CI: $2.77-$ $3.17)$ and $5.70 \pm 2.60 \mathrm{~mm}$ (95\% CI: 4.76-6.64), $3.03 \pm 1.04 \mathrm{~mm}$ (95\% CI: 2.53-3.53), respectively. The differences of the mean maximum diameter and mean neck size between CTA and 3D-DSA were not statistically significant (C-CTA/3DDSA: $P=0.846,0.745$; L-CTA/3D-DSA: $P=0.642$, $0.708)$. In both groups, there were significant correlations for maximum aneurysm diameter and aneurysm neck diameter 
TABLE 4: The results of C-CTA and L-CTA in detection of various sizes of aneurysms.

\begin{tabular}{lcccccccc}
\hline \multirow{2}{*}{ Size of aneurysms } & \multicolumn{2}{c}{ C-CTA } & \multicolumn{2}{c}{ L-CTA } & \multicolumn{2}{c}{ Detection rate } & & Detection \\
number & $\begin{array}{c}\text { Missing } \\
\text { number }\end{array}$ & $\begin{array}{c}\text { Detection } \\
\text { number }\end{array}$ & $\begin{array}{c}\text { Missing } \\
\text { number }\end{array}$ & C-CTA & L-CTA & Chi-square value & $P$ value \\
\hline$\geq 5 \mathrm{~mm}$ & 62 & 1 & 59 & 1 & $98.41 \%$ & $98.33 \%$ & $\ldots$ & 1.0 \\
$3 \sim 5 \mathrm{~mm}$ & 60 & 3 & 49 & 2 & $95.24 \%$ & $96.08 \%$ & 0.000 & 1.0 \\
$<3 \mathrm{~mm}$ & 18 & 5 & 10 & 3 & $78.26 \%$ & $76.92 \%$ & 0.000 & 1.0 \\
\hline
\end{tabular}

measurement between CTA and 3D-DSA $(r=0.975, r=$ $0.954 ; r=0.940, r=0.931)$.

\section{Discussion}

At present, traditional DSA examination still remains the gold standard for detection of aneurysm, but it has several limits: it is invasive and time-consuming, the operating technology is complicated, and it has about $1-2.3 \%$ of surgical complications [17]. As a noninvasive examination, the value of cerebral CTA in diagnosis of intracranial aneurysm is widely accepted by clinic, owing to its features of being noninvasive, simple, rapid, and accurate. But it also has the potential to lead to an increase in radiation dose, owing to the routine use of thinner sections and the extended volume of acquisition. Previous literature reported that the effective dose produced by one routine brain CT scan is $0.9-4.0 \mathrm{mSv}$, while the radiation dose which resulted from cerebral CTA is much greater. If a dual-phase or a subtraction angiography scan is performed, the radiation dose is 2 to 3 times more than that of single phase cerebral CTA scan [18]. Therefore, concerns regarding a reduction in radiation dose have been recently raised during cerebral CTA acquisitions [19].

Cerebral CTA scan with lower tube voltage can greatly reduce the radiation dose. In our study, reduction of tube voltage from $120 \mathrm{KV}$ to $100 \mathrm{KV}$ while keeping other parameters the same led to an approximate $35 \%$ decrease in radiation dose. Sun et al. [12] suggested that a tube voltage of $80 \mathrm{KV}$ resulted in an approximate $69.73 \%$ reduction in radiation dose compared with radiation exposure at $120 \mathrm{KV}$. However, the main drawback of the low tube voltage technique is the increase of image noise caused by the reduced photon flux. Some studies [11] suggested that increase of noise will affect the appearance of small diameter arteries, especially posterior circulation arterial aneurysms. In our study, there were no significant differences in objective and subjective image qualities between two groups. Furthermore, the aneurysm detectable rate of L-CTA group (41/48) is higher than that of C-CTA group (38/49). The analysed reasons are as follows: (1) the cerebral CTA scan with low tube voltage can significantly increase the vascular enhancement value, so it can improve the appearance of aneurysm itself; (2) the contrast between aneurysm and the surrounding parenchyma can be also increased, which will make up for the noise increase to a certain extent.

Without degradation of image quality, the value of low tube voltage CTA scan in detection of intracranial aneurysms was high. With the results of 3D-DSA as "gold standard," the sensitivity, specificity, and accuracy of C-CTA and LCTA in diagnosis of aneurysms were $93.96 \%, 99.83 \%$, and $99.38 \%$ and $95.16 \%, 99.72 \%$, and $99.42 \%$, respectively. There were no significant differences in sensitivity, specificity, and accuracy between the C-CTA and L-CTA groups, which were consistent with previous results [12].

Compared with the conventional DSA, CT image has relatively low spatial resolution. Therefore, the diagnostic accuracy of microaneurysms is much lower. Previous literatures $[20,21]$ reported that the CTA diagnostic accuracy of aneurysm with diameter $<3 \mathrm{~mm}$ was $74.1 \%$ to $84.0 \%$. In our study, the CTA diagnostic accuracy of aneurysms with diameter $\geq 3 \mathrm{~mm}$ achieved above $95 \%$, while the $<3 \mathrm{~mm}$ aneurysms' detection rate was low (C-CTA: 78.26\%; L-CTA: $76.92 \%)$. However, there were no statistically significant differences in diagnostic accuracy between the two groups. This indicates that the ability of detection of microaneurysm will not be affected by lower tube voltage CTA scan. The reasons of false-positive and false-negative results were based on diagnostic experience and improper operation or were due to aneurysm's secluded location or were affected by vascular malformation. The missed aneurysms in both CCTA and L-CTA groups can all be revealed on CTA images after retrospective reconstruction and analysis.

In conclusion, as an effective technique of reducing CT radiation dose, cerebral CTA with low tube voltage provides qualified image quality and high diagnostic value in detection of intracranial aneurysms. Therefore, it is a promising alternative for evaluating intracranial aneurysms and should be recommended as a routine scan method.

\section{Conflict of Interests}

The authors declare that there is no conflict of interests regarding the publication of this paper.

\section{References}

[1] L. H. Phillips II, J. P. Whisnant, W. M. O’Fallon, and T. M. Sundt Jr., "The unchanging pattern of subarachnoid hemorrhage in a community," Neurology, vol. 30, no. 10, pp. 1034-1040, 1980.

[2] F. Ildan, M. Tuna, T. Erman et al., "Prognosis and prognostic factors for unexplained subarachnoid hemorrhage: review of 84 cases," Neurosurgery, vol. 50, no. 5, pp. 1015-1025, 2002.

[3] C. E. Lovelock, G. J. E. Rinkel, and P. M. Rothwell, "Time trends in outcome of subarachnoid hemorrhage: populationbased study and systematic review," Neurology, vol. 74, no. 19, pp. 1494-1501, 2010. 
[4] H. B. Locksley, "Natural history of subarachnoid hemorrhage, intracranial aneurysms and arteriovenous malformations. Based on 6368 cases in the cooperative study," Journal of Neurosurgery, vol. 25, no. 2, pp. 219-239, 1966.

[5] B. Stegmayr, M. Eriksson, and K. Asplund, "Declining mortality from subarachnoid hemorrhage: changes in incidence and case fatality from 1985 through 2000," Stroke, vol. 35, no. 9, pp. 20592063, 2004

[6] A. M. H. Sailer, B. A. J. M. Wagemans, P. J. Nelemans, R. de Graaf, and W. H. van Zwam, "Diagnosing intracranial aneurysms with $\mathrm{mr}$ angiography: systematic review and metaanalysis," Stroke, vol. 45, no. 1, pp. 119-126, 2014.

[7] A. Ringelstein, U. Lechel, D. M. Fahrendorf, J. C. Altenbernd, M. Forsting, and M. Schlamann, "Radiation exposure in perfusion CT of the brain," Journal of Computer Assisted Tomography, vol. 38, no. 1, pp. 25-28, 2014.

[8] D. Beitzke, F. Wolf, G. Edelhauser et al., "Computed tomography angiography of the carotid arteries at low $\mathrm{kV}$ settings: a prospective randomised trial assessing radiation dose and diagnostic confidence," European Radiology, vol. 21, no. 11, pp. 2434-2444, 2011.

[9] G. A. Zamboni, S. Guariglia, A. Bonfante, C. Martino, C. Cavedon, and R. P. Mucelli, "Low voltage CTPA for patients with suspected pulmonary embolism," European Journal of Radiology, vol. 81, no. 4, pp. e580-e584, 2012.

[10] A. Waaijer, M. Prokop, B. K. Velthuis, C. J. G. Bakker, G. A. P. de Kort, and M. S. van Leeuwen, "Circle of Willis at CT angiography: dose reduction and image quality - reducing tube voltage and increasing tube current settings," Radiology, vol. 242, no. 3, pp. 832-839, 2007.

[11] B. B. Ertl-Wagner, R.-T. Hoffmann, R. Bruning et al., "Multidetector row CT angiography of the brain various kilovoltage settings," Radiology, vol. 231, no. 2, pp. 528-535, 2004.

[12] G. Sun, J. Ding, Y. Lu et al., "Comparison of standard- and low-tube voltage 320-detector row volume CT angiography in detection of intracranial aneurysms with digital subtraction angiography as gold standard," Academic Radiology, vol. 19, no. 3, pp. 281-288, 2012.

[13] M. Kidoh, T. Nakaura, T. Ogata et al., "Subtracted 3D CT angiography for the evaluation of intracranial aneurysms in 256-slice multidetector CT: usefulness of the $80-\mathrm{kVp}$ plus compact contrast medium bolus protocol," European Radiology, vol. 23, no. 11, pp. 3012-3019, 2013.

[14] L.-J. Zhang, S.-Y. Wu, J.-B. Niu et al., "Dual-energy CT angiography in the evaluation of intracranial aneurysms: image quality, radiation dose, and comparison with 3D rotational digital subtraction angiography," American Journal of Roentgenology, vol. 194, no. 1, pp. 23-30, 2010.

[15] M. V. Jayaraman, W. W. Mayo-Smith, G. A. Tung et al., "Detection of intracranial aneurysms: multi-detector row CT angiography compared with DSA," Radiology, vol. 230, no. 2, pp. 510518,2004

[16] S. H. Menzel and D. Teunen, "Guidelines on radiation dose on the patient," European Guidelines on Quality Criteria or Computed Tomography, p. 32, 2006.

[17] H. J. Cloft, G. J. Joseph, and J. E. Dion, "Risk of cerebral angiography in patients with subarachnoid hemorrhage, cerebral aneurysm, and arteriovenous malformation: a meta-analysis," Stroke, vol. 30, no. 2, pp. 317-320, 1999.

[18] D. J. Brenner and E. J. Hall, "Computed tomography-an increasing source of radiation exposure," The New England Journal of Medicine, vol. 357, no. 22, pp. 2277-2284, 2007.
[19] Z.-L. Li, H. Li, K. Zhang et al., "Improvement of image quality and radiation dose of CT perfusion of the brain by means of low-tube voltage (70 KV)," European Radiology, vol. 24, no. 8, pp. 1906-1913, 2014.

[20] D. Y. Yoon, K. J. Lim, C. S. Choi, B. M. Cho, S. M. Oh, and S. K. Chang, "Detection and characterization of intracranial aneurysms with 16-channel multidetector row CT angiography: a prospective comparison of volume-rendered images and digital subtraction angiography," American Journal of Neuroradiology, vol. 28, no. 1, pp. 60-67, 2007.

[21] S. Dammert, T. Krings, E. Ueffing et al., "Detection of intracranial aneurysms with multislice CT: comparison with conventional angiography," Neuroradiology, vol. 46, no. 6, pp. 427-434, 2004. 


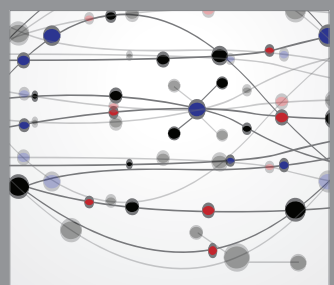

The Scientific World Journal
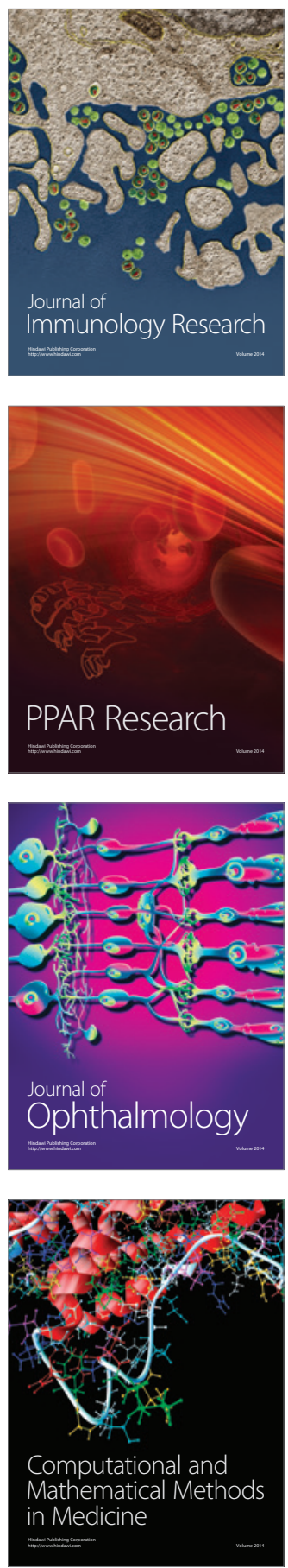

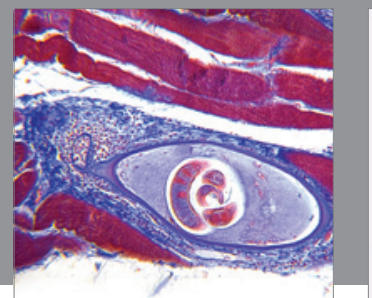

Gastroenterology

Research and Practice
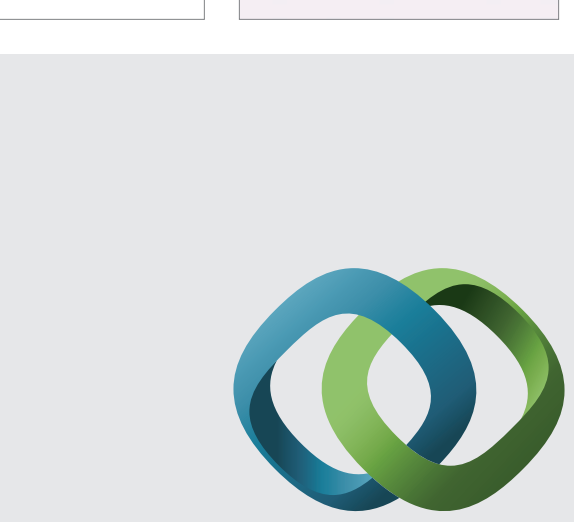

\section{Hindawi}

Submit your manuscripts at

http://www.hindawi.com
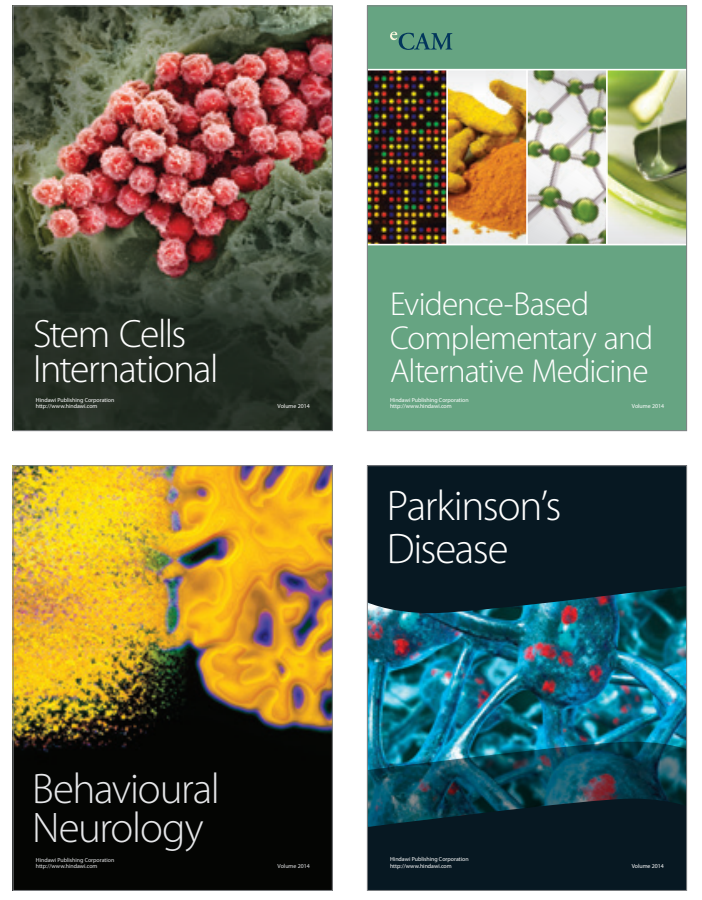
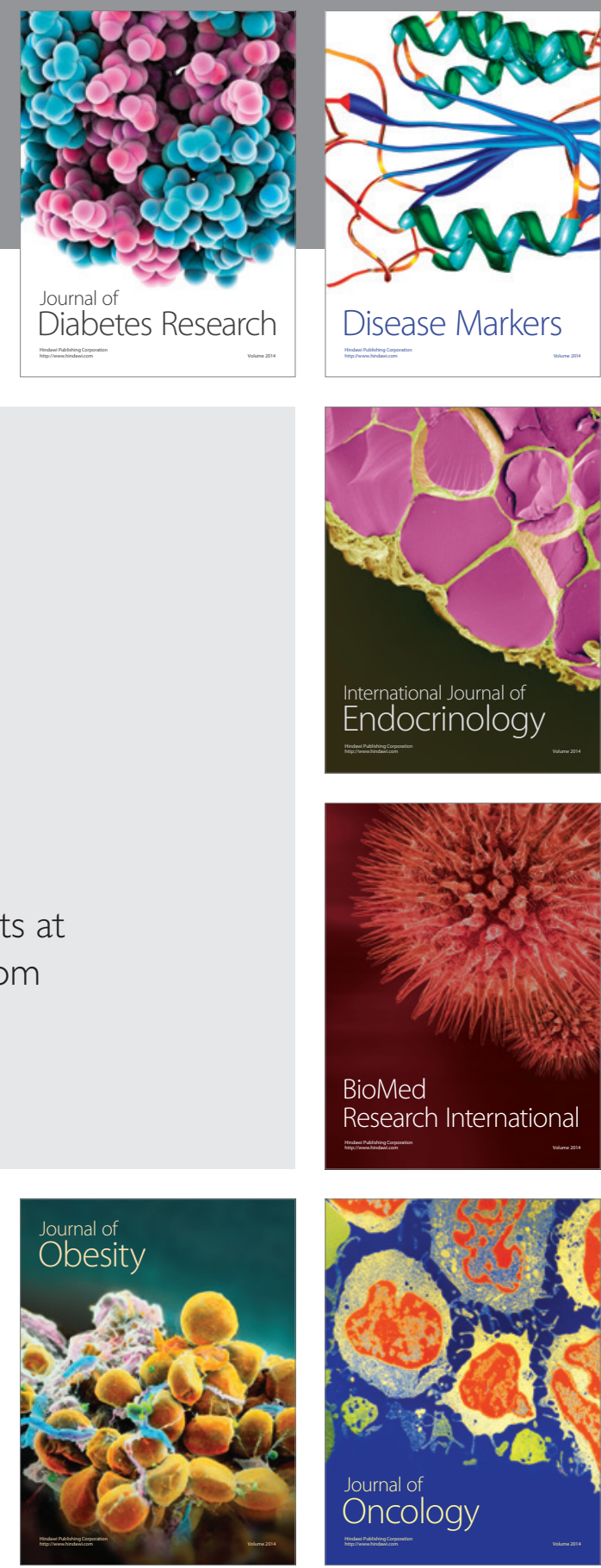

Disease Markers
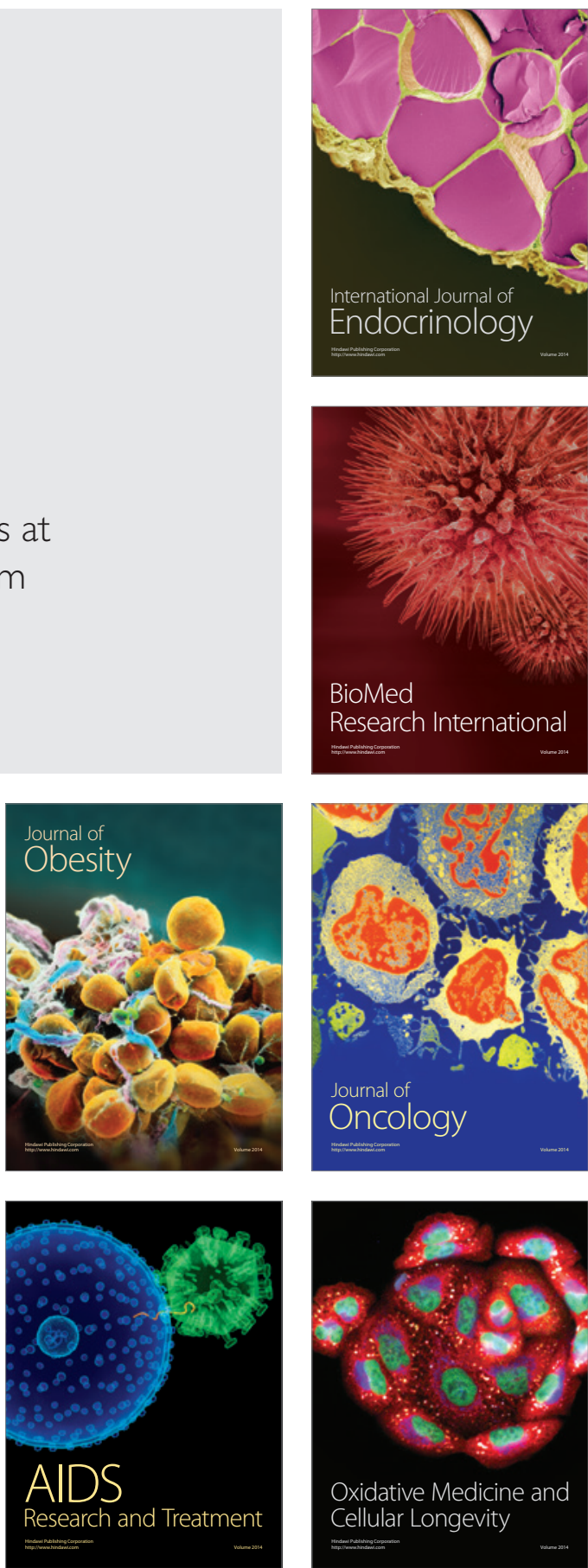